\title{
COCRYSTALS: A COMPLETE REVIEW ON CONVENTIONAL AND NOVEL METHODS OF ITS FORMATION AND ITS EVALUATION
}

\author{
BIJAY KUMAR YADAV ${ }^{1}$, ATIF KHURSHEED ${ }^{2}$, RATTAN DEEP SINGH ${ }^{2 *}$
}

${ }^{1}$ Deparment of School of Pharmaceutical Sciences, Lovely Professional University, Phagwara, Punjab, India. ${ }^{2}$ Department of School of Bioengineering and Biosciences, Lovely Professional University, Phagwara, Punjab, India. Email: drrattandeep@gmail.com

Received: 17 April 2019, Revised and Accepted: 01 June 2019

\section{ABSTRACT}

The active moiety with poor solubility is posing a challenge in drug development which may reduce the effectiveness in patients when administered orally. Cocrystal formation is one of the latest approaches for improving the various parameters of a drug molecule such as solubility, melting point, pharmacokinetic, pharmacodynamic, and bioavailability. Cocrystals are crystalline single state materials composed of two or more than two different molecular amalgams held together in a fixed stoichiometric ratio. There are various techniques used for the preparation of cocrystals such as solvent evaporation, grinding, and cooling crystallization. The quantitative and qualitative aspects of these cocrystals are evaluated using various validated instruments such as nuclear magnetic resonance, powder X-ray diffraction, and differential scanning calorimetry.

Keywords: Cocrystals, Coformers, Bioavailability, Crystallization, Grinding, Solubility.

(C) 2019 The Authors. Published by Innovare Academic Sciences Pvt Ltd. This is an open access article under the CC BY license (http://creativecommons. org/licenses/by/4. 0/) DOI: http://dx.doi.org/10.22159/ajpcr.2019.v12i7.33648

\section{INTRODUCTION}

Cocrystal concept of the supramolecular chemistry is gaining wide interest of researchers from pharmaceutical, chemical sciences, and regulatory agencies. Pharmaceutical cocrystal engineering has emerged as a new era in the field of medicine in developing a new moiety with improved solubility, dissolution, bioavailability, micrometric properties, and pharmacokinetic and pharmacodynamic properties of a drug. Cocrystals are crystalline and unagitated solid materials of two or more molecules held together within the same crystal lattice. However, pharmaceutical cocrystals are described as crystalline single state materials composed of two or more than two different molecular amalgams held together in a fixed stoichiometric ratio. The frequently accepted definition of pharmaceutical cocrystals, which emerged as a broad consensus opinion at the Indo-US meeting in 2011 and subsequently published in crystal growth and design is reproduced: "Cocrystals are solids crystalline single-phase materials composed of two or more different molecular and/or ionic compounds generally in a stoichiometric ratio which are neither solvates nor simple salts. Thus, it is a multiple component crystal modified by intermolecular interaction such as hydrogen bonding, van der Waals force, $\pi-\pi$ interactions, and halogen bond between an active pharmaceutical ingredient (drug) and conformer [1]. This cocrystal possesses a neoteric property than its parent drug molecule such as change in melting point, dissolution rate, micrometric properties, physiochemical properties, stability, permeability, and also, other various parameters of a drug moiety $(4 \mathrm{H})$. Hence, they can be easily patentable due to its novelty, nonobvious invention. Cocrystals are multicomponent crystals comprising salts, solvates, clathrates, inclusion crystals, and hydrates. In solvates, one component is liquid at room temperature, whereas in cocrystals, both components are solid at room temperature [2]. The major steps involved for supramolecular synthon determination for the preparation of cocrystals are as

Identification of important functional groups in the active pharmaceuticals ingredients (API) or moiety.

Insertion of functional groups in a systemic way in the Cambridge structural database to select and identify most potent coformer.

Select an appropriate technique for the cocrystal synthesis.
Perform various crystallization screening processes [3].

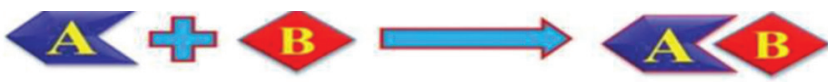

$\mathrm{A}=$ Active Pharmaceutical ingredients

$\mathrm{B}=$ Coformer

$\mathrm{AB}=$ Cocrystal

In 1844, Friedrich Wohler first carried out isolation of a cocrystal between quinone and hydroquinone. The concept of supramolecular synthons was first discovered by Gautam Desi Raju in 1955 which is considered as a key point in the study of crystal engineering for the synthesis of a new molecular entity. The US-FDA first approved a pharmaceutical ionic cocrystal drug in accordance with its cocrystal guidance paper for drug substance in 2012.

Examples of cocrystal drug in the market

1. Ernesto an antihypertensive drug (monosodium sacubitril+disodium valsartan+water) launched by Novartis for treatment of chronic heart failure in 2015

2. Lexapro (Escitalopram oxalate), an antidepressant drug (protonated escitalopram cations+water+oxalate dianion and deprotonated oxalic acid) approved in 2009

3. Ertugliflozin, an antidiabetic drug (5-oxo-proline+escitalopram)

4. Depakote (valproate sodium cocrystal in combination with valproic acid), most frequently used drug for the treatment of manic depression and seizure. It is one of the latest developments in the cocrystal formulation of a drug [4]. In general, the bond formation takes place between the adjacent moiety of a drug molecule leading to generation of new molecular compound with distinct properties $(3 \mathrm{~h})$

5. Baicalein and nicotinamide cocrystal-enhanced oral BA of 2.5-fold peak plasma concentration and 2.80-fold higher area under curve in rats

6. Hydrochlorothiazide-nicotinic acid cocrystal-enhanced solubility.

\section{TECHNIQUES OF COCRYSTAL FORMATION}

With the advancement in drug development, various methods are being used for the preparation of multicomponent solid forms such 


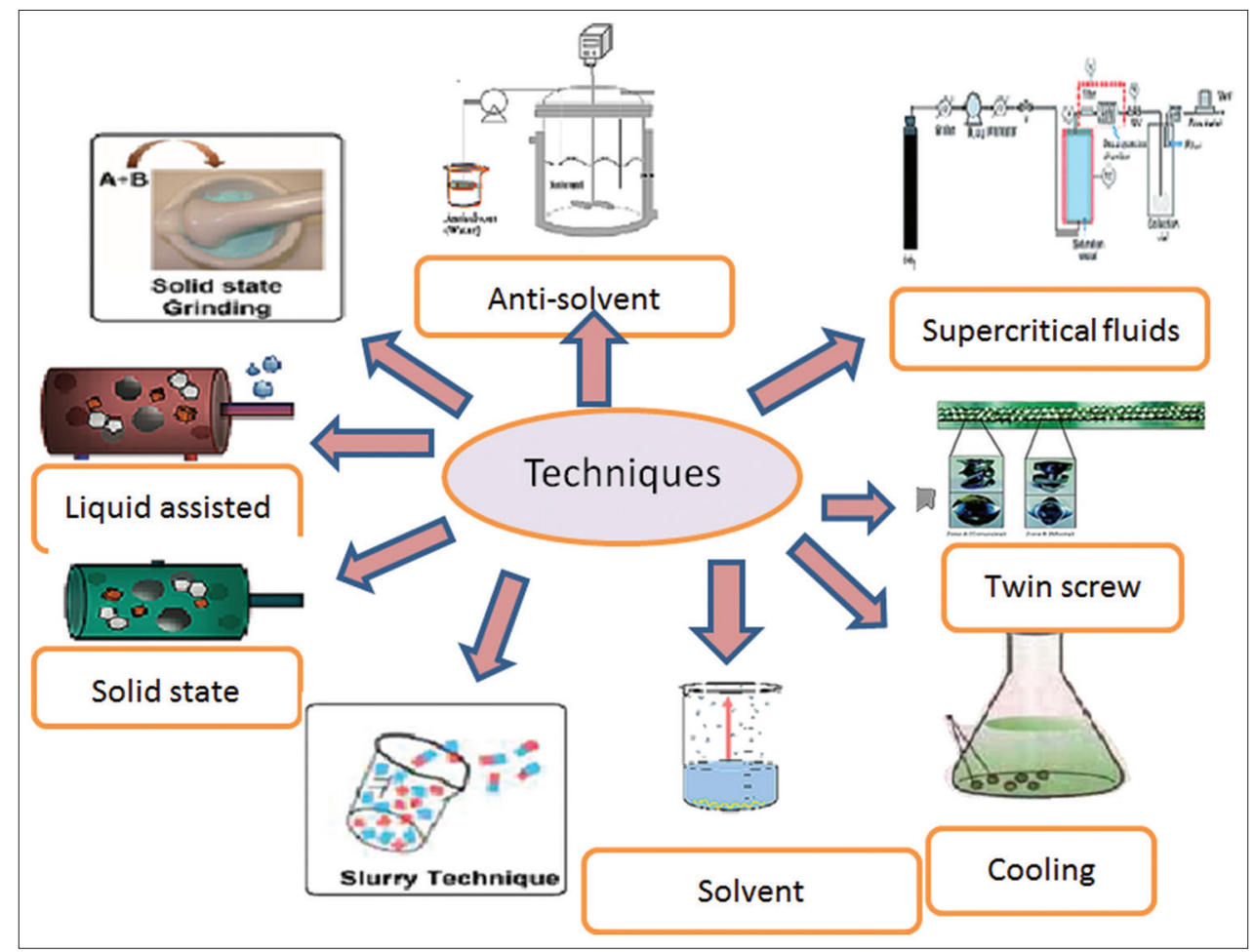

Fig. 1: Techniques of cocrystal formation [5]

as cocrystals, cosolvates, coamorphous, polymorphs, hydrates/salts, and eutectics. Solvent selection, API and coformers are the important parameters for such preparations. The various kinds of methods that are most commonly used are:

\section{Solid-based technique}

It generally includes solid phase grinding, melt extrusion, and melts crystallization. In this method, API and coformer are melted and mixed together, resulting in the cocrystal formation in a fixed stoichiometric ratio. It is basically not suitable for thermolabile moiety, but it is easy, scalable, and continuous process.

\section{Grinding method}

It is one of the mostly used techniques for the cocrystal formation from the last few years. It is basically of 2 types: (a) dry grinding method and (b) wet grinding method.

\section{Dry grinding method}

It is most widely and commonly used technique for cocrystal formation, in which API and coformers are mingled in a stoichiometric proportion using mortar and pestle. This method is simple, easy to perform, ecofriendly, and highly productive but is mechanical and timeconsuming. Nowadays, planetary milling systems are also available in a laboratory scale [6]. The examples of cocrystals prepared by this method are carbamazepine-nicotine amide, piracetam-citric acid, and piracetam-tartaric acid [7]. The liquid-assisted grinding method had greater cocrystal formation efficiency than piracetam-citric acid cocrystal by grinding technique [8], acyclovir-succinic acid cocrystal [9].

\section{Hot melt extrusion method}

In this technique, API and coformers are transferred into a fixed controlled temperature system where they are melted and form cocrystals of new moiety [10]. This technique is not suitable for thermolabile drugs because both drug and coformer should be mixed in a molten state. In this method, API and coformer are mixed in their molten state to enhance their surface contact without the use of solution (solvent), for example, pyrazinamide cocrystals [11].

\section{Liquid-based technique}

This technique mainly includes: Solvent evaporation [12], solvent drop grinding [13],liquid-assisted grinding [14], solution crystallization [15], cooling crystallization [16], supercritical fluids [17], slurry method [18], antisolvent method [19], reaction crystallization method [20], ultrasound-assisted solution technique [21], supercritical fluid atomization techniques [22], and spray drying techniques [23].

\section{SLOW EVAPORATION OF SOLVENT RESULTS COCRYSTAL FORMATION}

It is also known a solvent evaporation technique, in which a solution (solvent) is made to vaporize slowly. During the process of dissolution, the functional moiety in the API and coformer interchanges with each other to form new hydrogen bonds which is most widely used by many researchers, for example, glutaric acid cocrystals [24]. In this method, both API and coformers are dissolved with a continuous stirring in a boiling solvent until the final volume becomes small. This boiling solution is allowed to cool slowly to form cocrystals in either open air or in hot air oven [19], for example, theophylline-citric acid cocrystals [25].

In this technique, solvent dissolving coformers are selected and dissolved, and finally, drug is scattered into it by dispersion homogenizer. The solution is then mixed with a proper solvent for the precipitation of coformer into the drug indomethacin-saccharin cocrystal powders, carbamazepine-saccharin cocrystal [26].

\section{Liquid-assisted grinding method}

Liquid-assisted grinding is another commonly used method to form cocrystals. Besides providing faster rate of cocrystal formation than dry grinding, it is more reliable and suitable method as well. It is known to be ecofriendly method for industrial-level production due to less amount of solvent used. The process does not also depend on the temperature, and more importantly, it diminishes the chances of unwanted solvate formation, adefovir dipivoxil-glutaric acid cocrystal [27] and quercetinsuccinic acid cocrystal improves solubility and dissolution rate by 1.62 and 1.25 , respectively. 
Table 1: Solvent evaporation technique for various drugs

\begin{tabular}{|c|c|c|c|c|}
\hline S. No. & Drug & Coformer & Preparation technique & Property improved \\
\hline 1. & Fluoxetine $\mathrm{HCl}$ & $\begin{array}{l}\text { Benzoic acid } \\
\text { Fumaric acid } \\
\text { Succinic acid }\end{array}$ & Solvent evaporation & Dissolution rate \\
\hline 2. & Ibuprofen & 4,4-Dipyridyl Nicotinamide & Solvent evaporation & Solubility was increased by 62 times in suspension form \\
\hline 3. & Itraconazole & $\begin{array}{l}\text { Malic acid } \\
\text { Tartaric acid } \\
\text { Succinic acid }\end{array}$ & Solvent evaporation & $\begin{array}{l}\text { Enhanced dissolution and solubility rate as amorphous } \\
\text { form }\end{array}$ \\
\hline 5. & Piroxicam & Saccharin & Solvent evaporation & Improved dissolution rate and bioavailability \\
\hline 6. & Acyclovir & Nicotinamide & Solvent evaporation & Increase in dissolution rate \\
\hline
\end{tabular}

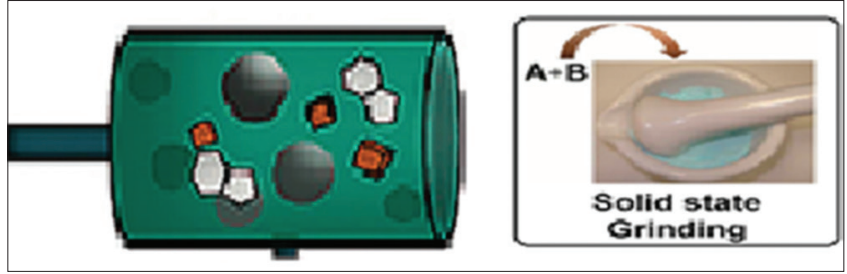

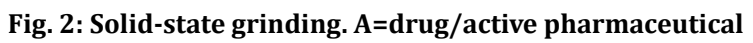
ingredients $B=$ Coformer/excipients

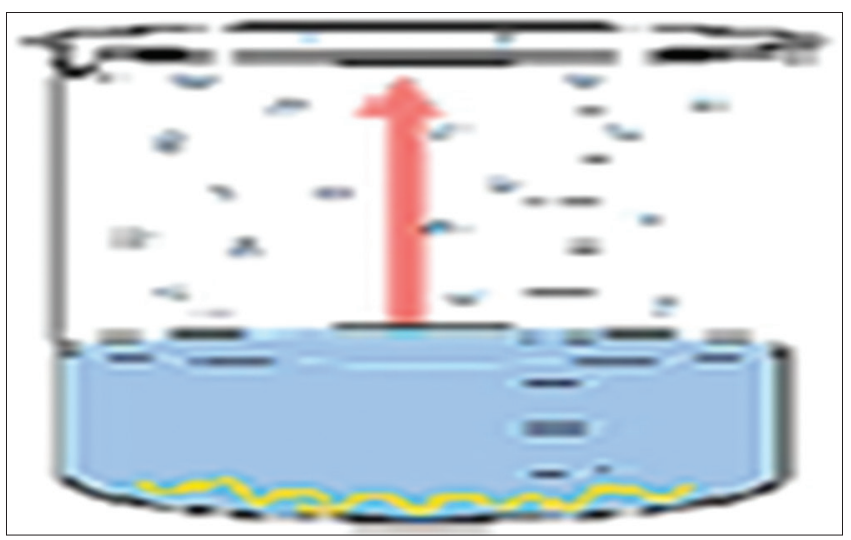

Fig. 3: Solvent evaporation technique

\section{Solvent drop grinding}

This method involves incorporation of API and coformer with the addition of suitable solvent. The solvent is added in drops with continuous stirring. The solvent used behaves as a catalyst which enhances crystal formation. This technique is also suitable for the synthesis of amorphous cocrystals. Example includes carbamazepinenicotinamide coamorphous crystals.

\section{Cooling crystallization method}

It is less frequently applied method for the cocrystal formation. It is generally slow and time-consuming process as compared to other techniques, for example, darunavir-succinic acid cocrystal. In this, there is an improvement in solubility, dissolution, and micrometric properties than its individual drug darunavir [28].

\section{Reaction crystallization method}

This technique is mainly employed for quick formation of cocrystals at the macroscopic and microscopic level at a room temperature in which cocrystallization and nucleation are based on their solubility and cocrystal formation. Drug and coformer are solvated separately in either methanol or other suitable solvent and finally mixed together for crystal formation under its solubility limit. To determine stoichiometry of the complex, precipitates are collected and examined by high-performance liquid chromatography [29].

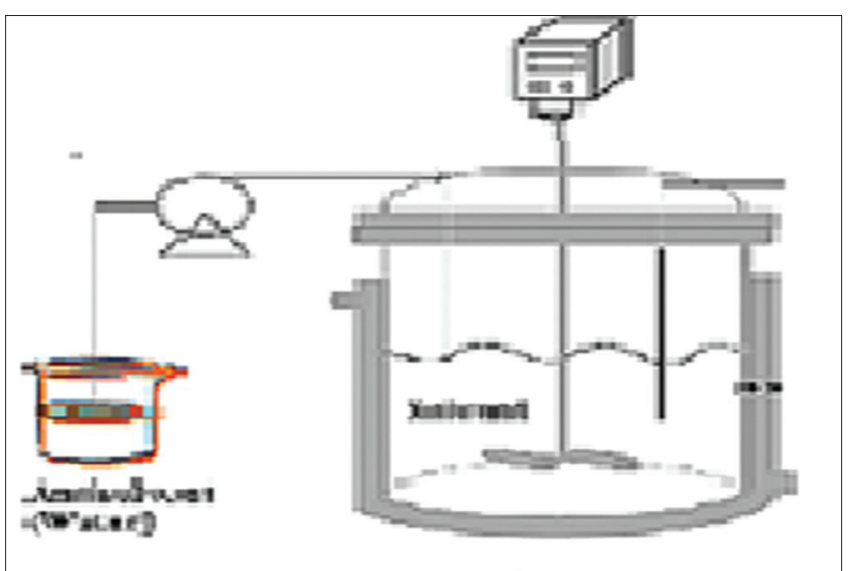

Fig. 4: Antisolvent method

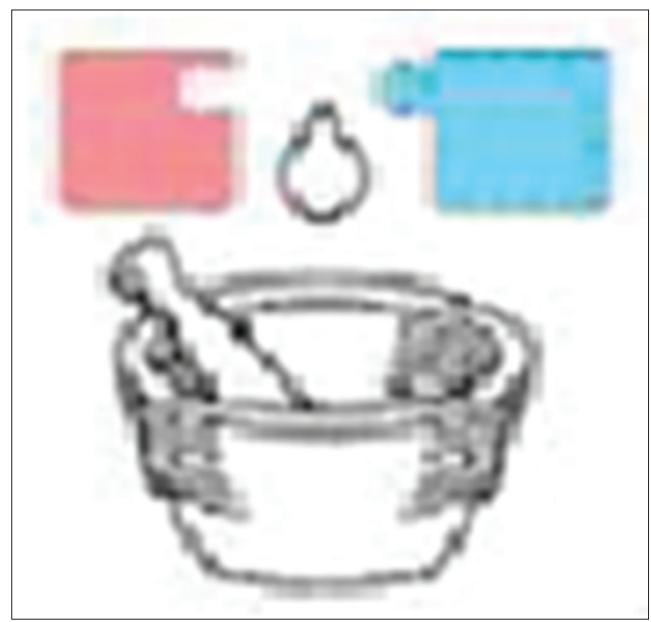

Fig. 5: Solvent drop method

Ultrasound-assisted solution method

This technique is used for the nanocrystal preparations in which drug and coformers are dissolved in an appropriate vehicle (solvent). Solution is placed in a sonicator to form turbid, and temperature is maintained to prevent fragmentation and degradation. Solution is kept overnight for solvent evaporation and cocrystal formation [30].

\section{Spray dying method}

It is very commonly employed method for the preparation of cocrystals because of its quick, continuous, and single step process. In this technique, solution containing API and coformer is allowed to evaporate over hot air stream. This technique is relevant to scale up and more user friendly, for example, sulfadimidine/4-aminosalicylic acid cocrystals [31]. 


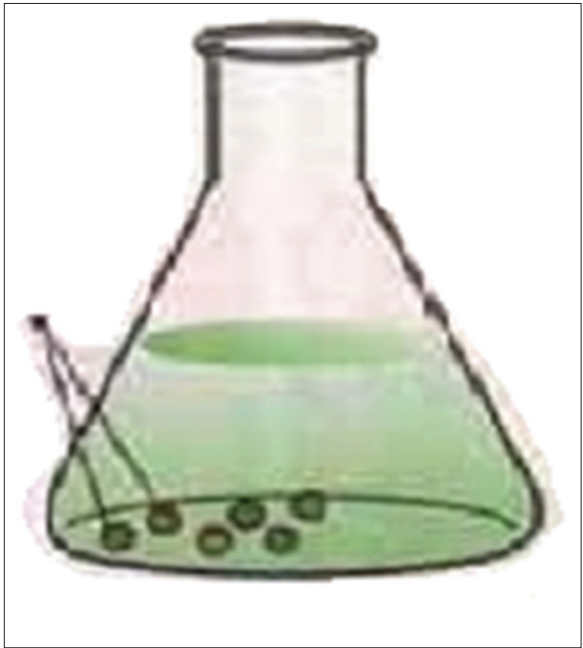

Fig. 6: Cooling crystallization

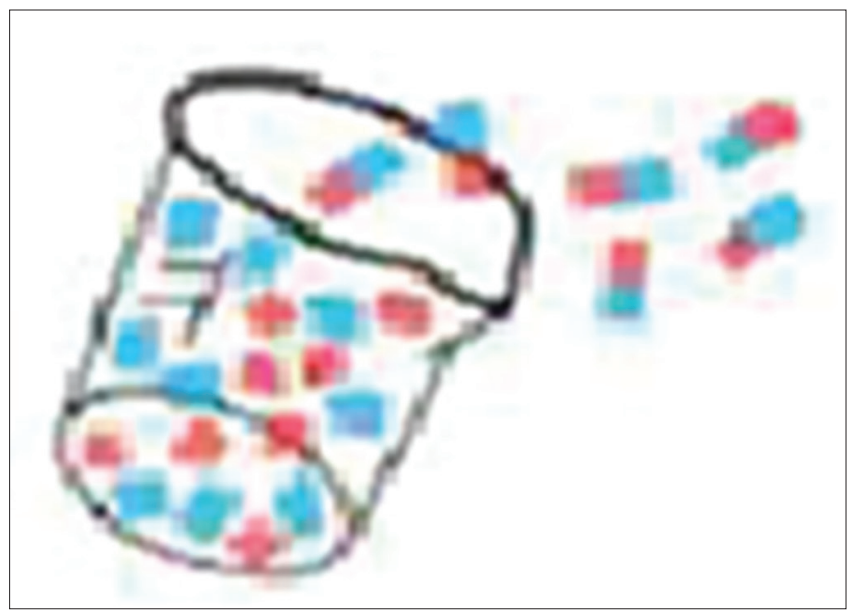

Fig. 7: Slurry technique

\section{Slurry method}

It is also one of the easiest techniques for the crystallization process where cocrystal formation takes place. The selected drug and coformer are dissolved into a suitable solvent forming a suspension and are finally stirred, filtered, and dried. Celecoxib-venlafaxine cocrystal (nonsteroidal anti-inflammatory drugs [NSAIDs] + antidepressant) drug was prepared and patented using slurry technique of crystallization. The celecoxib (BCS class II) and venlafaxine (BCS class I) solubility issue are overcome by the use of high solubility BCS class I drug [32].

In this technique, a slurry of API and coformer is prepared in a suitable solvent, stirred properly with a glass $\mathrm{rod} / \mathrm{magnetic}$ stirrer, and solvent is allowed to cool slowly at room temperature until cocrystal formation takes place, for example, acyclovir-succinic acid cocrystal [33], aspirin-4,4, dipyridyl cocrystals [34], and trimethoprimsulfamethoxazole cocrystals.

\section{Supercritical antisolvent technique}

This method is most useful technique for crystal preparation and to prevent the thermal degradation of compound. In this technique, solid sample is dissolved in a suitable solvent (organic or inorganic) which is injected into a supercritical fluid (under high pressure) resulting in a large decrease in solution density forming cocrystals [35]. The bioavailability of irbesartan was improved by this technique along with its dissolution rate, and Pluronic F127 was used as surfactant [30]. Indomethacin-saccharin cocrystals were first prepared by Gomes de Azevedo. Likewise, piracetam-salicylic acid cocrystals,
Table 2: List of coformers

\begin{tabular}{ll}
\hline S. No. & Ketoglutaric acid \\
\hline 1. & Maleic acid \\
2. & Glutaric acid \\
3. & Malonic acid \\
4. & Oxalic acid \\
5. & Adipic acid \\
6. & Camphoric acid \\
7. & 4-hydroxybenzoic acid \\
8. & Salicylic acid \\
9. & 1-hydroxy-2-naphthoic acid \\
10. & Ketoglutaric acid \\
\hline
\end{tabular}

diflunisal-nicotinamide cocrystals, paracetamol-dipicolinic acid, and naproxen-nicotinamide cocrystals are some of the examples prepared by this method. $\mathrm{CO}_{2}$ (non-polar compound) is the best supercritical fluid applied in the pharmaceutical fields due to its advantages such as non-toxic, non-flammable, economical, and easily available [36]

\section{EVALUATION AND IDENTIFICATION OF CO-CRYSTALS}

Fourier-transform infrared spectroscopy: It is widely used process for the prediction and determination of chemical conformation, intermolecular interactions, and communion study between API and coformers. This method is quick, nondestructive, prone to changes in molecular structure and can also detect a functional group.

Differential scanning calorimetry (DSC): It is used for the determination of cocrystal formation, determined by the existence of exothermic crest followed by endothermic crest in the DSC spectra [32]. The cocrystal formation is determined by the presence of crest (peaks) present in the compound. It is also useful for determining the melting point, polymorphic nature, glass temperature, heat of fusion, and exothermic or endothermic behavior of a compound or a molecule [37].

\section{Thermal gravimetry method}

This method is useful for determining the sample weight under the influence of temperature for a specific period of time. It gives exact drying temperature along the various reaction steps involved in the component. It is used for the prediction of stability, purity, compatibility, and solvates/hydrates forms of cocrystals [38].

\section{Terahertz time-domain spectroscopy}

This technique is similar to that of powder X-ray diffraction (PXRD) for the characterization and identification of cocrystals. It is useful for differentiating supramolecular structures, chiral, and racemic molecule present in a given sample [39]. Example includes cocrystals of theophylline with different conformers [40].

\section{Solid-state nuclear magnetic resonance (SSNMR)}

SSNMR is generally used for characterization and identification of various solid forms of pharmaceutical products including the cocrystals. The basic principle used in SSNMR is the nuclei shift by irradiation that differs it from the excipients. The common examples are 13C, 31P, $1 \mathrm{H}$, and $19 \mathrm{~F}$ [41]. By this quantitative and qualitative technique, we can determine the molar ratio of reaction mixture and type of hydrogen atom present in a given molecule $[42,43]$.

\section{PXRD}

It deals with the study of crystalline behavior of a powder or a drug sample. Bolla et al. carried-out analysis of acemetacin cocrystals using these analytical techniques. They revealed the crystalline cell dimension, purity, structure, and texture of this bulk sample [44].

\section{Dissolution studies}

It can be defined as "the quantity of drug substance that changes into a solution in a unit time in specific conditions of liquid/solid interface, solvent composition, and temperature." In-vitro dissolution study 
of any solid drug is carried out to evaluate the dissolution efficacy of formulated drug [45].

\section{Stability study}

It is also one of the potent parameters for the evaluations of cocrystals as it gives information about different climatic storage conditions and shelf life of the drug or drug products. There are various parameters that affect the stability of drug such as humidity, light, and temperature [46]

Hansen Solubility study: Hansen solubility parameter is one of the potent tools to predict the miscibility of a drug and coformer in crystal formation or with excipients/carriers. It can also predict compatibility of pharmaceutical materials and its study is also useful for the preformulation and formulation of tablets [47]. The cohesion energy is utilized to predict physiochemical properties such as melting point and solubility of a compound [48]. Cocrystals are held together by a weak hydrogen bonding and are miscible at the molecular level. In solubility study, different types of solvents are used such as water, buffer solutions of different $\mathrm{pH}$, stimulated intestinal fluid, and gastric fluid. It is one of the potent parameters of drug testing for drug development. The solution stability has been performed in various cocrystals such as carbamazepine/saccharin cocrystal, succinic acid cocrystal, indomethacin-saccharin cocrystals, and glutaric acid cocrystals.

\section{APPLICATIONS OF COCRYSTALS}

\section{Bioavailability}

It is a potent parameter to determine the amount of drug that reaches into the systemic circulation, and there is lesser number of animal studies on bioavailability of cocrystals. Cocrystals are developed to improve its bioavailability, solubility, permeability, dissolution, and other physiochemical properties of an API with use of coformers. e.g., indomethacin-saccharin cocrystals have improved bioavailability than its pure API drug, indomethacin cocrystals of glutaric acid and 2-[4-(4-chloro-2-fluorphenoxy) phenyl]-pyrimidine-4-carboxamide had improved oral bioavailability [49].

\section{Solubility}

Low solubility drug can be improved with cocrystal formation in which solubility is enhanced by a conformer of highly solubility. Example: Meloxicam-aspirin cocrystal a NSAID drug with low aqueous solubility and high permeability had slow onset of action ( $>2 \mathrm{~h}$ ). The issue was solved with cocrystal formulation in the form of faster dissolution, improved oral absorption, and quick/early onset of action. In this, aspirin was used as conformer [50]. The solubility issue of both drug ibuprofen and flurbiprofen was overcome with coformer nicotinamide forming cocrystal with each drug. Tableting behavior and moisture sorption increased dissolution rate by 8 -fold and 5 -fold, respectively. Likewise, AMG 517 cocrystal decreases the dose by increasing dissolution rate of active moiety. The solubility issue of itraconazole was overcome by forming a cocrystal with malic acid and dissolution rate was also improved [51].

\section{Stability}

Temozolomide (TMZ)-succinic acid, TMZ-malic acid, and TMZ-tartaric acid cocrystals, an anticancer drug, are known to be more stable at $\mathrm{pKa}$ 2-6 than the pure drug. With temperature $40^{\circ} \mathrm{C}$ and relative humidity $75 \%$, TMZ started degrading after a week except TMZ-succinic acid and TMZ-oxalic acid. This stability pattern was revealed using PXRD [52].

\section{Melting point}

It is the temperature at which solid material is at equilibrium with liquid phase [53]. The melting point of a drug is also related to its solubility, stability, tablet ability, flow property, and processability of a drug formulation. The cocrystal form of drug generally decreases the melting point than its pure drug and coformer. The melting point of hexamethylenebisacetamide (anticancer drug)-dicarboxylic acid cocrystals had reduced melting point than its pure drug along with the improvement in the physiochemical properties of a drug [54].

\section{Tablet stability}

The proper and elegant tablet formation is also one of the most potent parameters of a cocrystal development. The cocrystals of efavirenzadipic acid and cocrystals of efavirenz-lactic acid show 3-3.5-fold improvement in the solubility along with its tablet property [55]. The fenofibrate-nicotinamide cocrystals prepared with ethanol by solution technique had improved tableting property like plasticity and flowability [56].

\section{COFORMER}

Coformers are considered as inert, pharmacologically safe component for the formation of cocrystals. The selection of coformer is one of the most tedious tasks in cocrystal development and synthesis. They are generally used to enhance various properties of a drug such as micromeritics, stability, and dissolution pharmacological depending on the nature and category of drug to which they belong. It may be either a drug or excipient [56]. These coformers are therapeutically or pharmaceutically safe, inert, economical, and are easily available.

\section{TYPES OF COCRYSTALS}

\section{Molecular cocrystals}

These cocrystals contain only neutral components (coformers).

\section{Ionic cocrystals}

In ionic cocrystals, the dominant interaction has an electrostatic nature with the possible additional contributions of hydrogen bonds between commondonor andacceptor $(\mathrm{OH}, \mathrm{NH}, \mathrm{O}, \mathrm{N}$, etc.). The best example of this is ionic cocrystals of sodium chloride with carbohydrates [57]. Childs et al. studied on the organic cation halides based on ionic cocrystals and fluoxetine hydrochloride ICCs with a group of carboxylic acid coformers was also invented by him. Tetracycline ICCs with improved performance, saxagliptin hydrochloride with

improved stability, and streptomycin acid salts with alkaline earth metals are some of examples of ICCs [58].

\section{Multidrug cocrystals}

These are crystalline solid form of materials made of two or more than two therapeutic molecules in a stoichiometric ratio. These are also known as drug-drug cocrystals. It has become a newer approach for drug development [59].

Several crystal structure prediction programs were designed to find the crystal structure of an organic molecule, starting from the chemical diagram. However, the existence of polymorphism, the appearance of different crystal structures containing only the same molecule, immediately shows that some crystal structures are not the thermodynamically most stable. Molecules often they crystallize first in a metastable polymorph and then can be transferred into stable form [60].

\section{CONCLUSION}

Crystallization is one the noble approaches for improving the various parameters of active pharmaceutical ingredients such as stability, solubility, micrometrics properties, dissolution bioavailability, and pharmacokinetic properties by proper selection with coformers. The selection of coformers must be as per the United State Food and Drug Administration guidelines which do not produce any toxic effect with the drug. The techniques used for the preparation of cocrystals must be easy, safe, saleable, ecofriendly, and productive. Cocrystals also provides useful parameters such as improvement in permeability of poorly soluble drugs and better tableting property in case of poor tablet forming drugs.

\section{AUTHORS' CONTRIBUTIONS}

The author declares that all the authors listed in the paper have contributed to the literature review for this paper.

\section{CONFLICTS OF INTEREST}

No conflicts of interest are associated with this work. 


\section{REFERENCES}

1. Aakeröy CB, Champness NR, Janiak C. Recent advances in crystal engineering. CrystEngComm 2010;12:22-43.

2. Almarsson $\mathrm{O}$, Zaworotko MJ. Crystal engineering of the composition of pharmaceutical phases. Do pharmaceutical co-crystals represent a new path to improved medicines? Chem Commun (Camb) 2004;:1889-96.

3. Diniz SL, Souza MS, Ellena J. Crystal engineering applied to the development of novel pharmaceutical solid forms with improved bioavailability: The co crystals case. Adv Bioequi Bioavail 2018;1:1-3.

4. Bolla G, Nangia A. Pharmaceutical cocrystals: Walking the talk. Chem Commun (Camb) 2016;52:8342-60.

5. Chadha R, Bhalla Y, Vashisht MK, Chadha K. Cocrystallization in nutraceuticals. In: Recrystallization in Materials Processing. S.1.: IntechOpen; 2015.

6. Bysouth SR, Bis JA, Igo D. Cocrystallization via planetary milling: Enhancing throughput of solid-state screening methods. Int J Pharm 2011;411:169-71.

7. Rehder S, Klukkert M, Löbmann KA, Strachan CJ, Sakmann A, Gordon $\mathrm{K}$, et al. Investigation of the formation process of two piracetam cocrystals during grinding. Pharmaceutics 2011;3:706-22.

8. Rehder S, Klukkert M, Löbmann KA, Strachan CJ, Sakmann A, Gordon $\mathrm{K}$, et al. Investigation of the formation process of two piracetam cocrystals during grinding. Pharmaceutics 2011;3:706-22.

9. Jung S, Choi I, Kim I. Liquid-assisted grinding to prepare a cocrystal of adefovir dipivoxil thermodynamically less stable than its neat phase. Crystals 2015;5:583-91.

10. Dhumal RS, Kelly AL, York P, Coates PD, Paradkar A. Cocrystalization and simultaneous agglomeration using hot melt extrusion. Pharm Res 2010;27:2725-33.

11. Boksa K, Otte A, Pinal R. Matrix-assisted cocrystallization (MAC) simultaneous production and formulation of pharmaceutical cocrystals by hot-melt extrusion. J Pharm Sci 2014;103:2904-10.

12. Manin AN, Voronin AP, Drozd KV, Manin NG, Bauer-Brandl A, Perlovich GL. Cocrystal screening of hydroxybenzamides with benzoic acid derivatives: A comparative study of thermal and solution-based methods. Eur J Pharm Sci 2014;65:56-64.

13. Kumar S. Pharmaceutical cocrystals: An overview. Indian J Pharm Sci 2018:79:858-71.

14. Maroyi A. A comparative study of medicinal plants used in rural areas of Namibia and Zimbabwe. Indian J Indian Knowl 2015;14:401-6.

15. Mirza S, Miroshnyk I, Heinämäki J, Yliruusi J. Co-crystals: An emerging approach for enhancing properties of pharmaceutical solids. Dosis 2008;24:90-6.

16. Oberoi LM, Alexander KS, Riga AT. Study of interaction between ibuprofen and nicotinamide using differential scanning calorimetry, spectroscopy, and microscopy and formulation of a fast-acting and possibly better ibuprofen suspension for osteoarthritis patients. J Pharm Sci 2005;94:93-101

17. Remenar JF, Morissette SL, Peterson ML, Moulton B, MacPhee JM, Guzmán HR, et al. Crystal engineering of novel cocrystals of a triazole drug with 1,4-dicarboxylic acids. J Am Chem Soc 2003;125:8456-7.

18. Basavoju S, Boström D, Velaga SP. Pharmaceutical cocrystal and salts of norfloxacin. Cryst Growth Des 2006;6:2699-708.

19. Childs SL, Hardcastle KI. Cocrystals of piroxicam with carboxylic acids. Cryst Growth Des 2007;7:1291-304.

20. Nawatila R, Nuneik WA, Siswodhiardjo S, Setyawann D. Prepartion of acyclovir nicotinamide co-crystal with solvent evaporation techniquewith variation of the solvent. Asian J Pharm Clin Res 2017;10:283-7.

21. Chun NH, Lee MJ, Song GH, Chang KY, Kim CS, Choi GJ. Combined anti-solvent and cooling method of manufacturing indomethacinsaccharin (IMC-SAC) co-crystal powders. J Cryst Growth 2014;408:112-8.

22. Bagde SA, Upadhye KP, Dixit GR, Bakhle SS. Formulation and evaluation of co-crystals of poorly water soluble drug. Int J Pharm Res 2016;7:4988-97.

23. Sugandha K, Kaity S, Mukherjee S, Isaac J, Ghosh A. Solubility enhancement of ezetimibe by a cocrystal engineering technique. Cryst Growth Des 2014;14:4475-86.

24. Aher S, Dhumal R, Mahadik K, Paradkar A, York P. Ultrasound assisted cocrystallization from solution (USSC) containing a non-congruently soluble cocrystal component pair: Caffeine/maleic acid. Eur J Pharm Sci 2010;41:597-602.

25. Grossjohann C, Serrano DR, Paluch KJ, O’Connell P, Vella-Zarb L, Manesiotis $\mathrm{P}$, et al. Polymorphism in sulfadimidine/4-aminosalicylic acid cocrystals: Solid-state characterization and physicochemical properties. J Pharm Sci 2015;104:1385-98

26. Salaman CR, Ces SV, Tesson N, Castano MT. Co-Crystals of Venlafaxine and Celecoxib. Europe: Google Patents; 2013.

27. Rahman F, Winantari AN, Setyawan D, Siswandono S. Comparison study of grinding and slurry method on physicochemical characteristic of aclycovir-succinic acid cocrystal. Asian J Pharm Clin Res 2017;10:153-8.

28. Qiao N, Li M, Schlindwein W, Malek N, Davies A, Trappitt G. Pharmaceutical cocrystals: An overview. Int J Pharm 2011;419:1-11.

29. Rantakylä M, Jäntti M, Aaltonen O, Hurme M. The effect of initial drop size on particle size in the supercritical antisolvent precipitation (SAS) technique. J Supercrit Fluids 2002;24:251-63.

30. Adeli E. The use of supercritical anti-solvent (SAS) technique for preparation of Irbesartan-Pluronic ${ }^{\circledR}$ F-127 nanoparticles to improve the drug dissolution. Powder Technol 2016;298:65-72.

31. Kalani M, Yunus R. Application of supercritical antisolvent method in drug encapsulation: A review. Int J Nanomedicine 2011;6:1429-42.

32. Wu TK, Lin SY, Lin HL, Huang YT. Simultaneous DSC-FTIR microspectroscopy used to screen and detect the co-crystal formation in real time. Bioorg Med Chem Lett 2011;21:3148-51.

33. Yamashita H, Hirakura Y, Yuda M, Terada K. Coformer screening using thermal analysis based on binary phase diagrams. Pharm Res 2014;31:1946-57.

34. Jiang L, Huang Y, Zhang Q, He H, Xu Y, Mei X. Preparation and solidstate characterization of dapsone drug-drug co-crystals. Cryst Growth Des 2014;14:4562-73.

35. El-Gizawy SA, Osman MA, Arafa MF, El Maghraby GM. Aerosil as a novel co-crystal co-former for improving the dissolution rate of hydrochlorothiazide. Int J Pharm 2015;478:773-8.

36. Parrott EP, Zeitler JA, Friščić T, Pepper M, Jones W, Day GM, et al. Testing the sensitivity of terahertz spectroscopy to changes in molecular and supramolecular structure: a study of structurally similar cocrystals. Cryst Growth Des 2009;9:1452-60.

37. Stevens JS, Byard SJ, Schroeder SL. Salt or co-crystal? Determination of protonation state by X-ray photoelectron spectroscopy (XPS). J Pharm Sci 2010;99:4453-7.

38. van der Wel PC. New applications of solid-state NMR in structural biology. Emerg Top Life Sci 2018;2:57-67.

39. Kijac AZ, Li Y, Sligar SG, Rienstra CM. Magic-angle spinning solidstate NMR spectroscopy of nanodisc-embedded human CYP3A4. Biochemistry 2007;46:13696-703.

40. Bolla G, Chernyshev V, Nangia A. Acemetacin cocrystal structures by powder X-ray diffraction. IUCrJ 2017;4:206-14.

41. Padrela L, de Azevedo EG, Velaga SP. Powder X-ray diffraction method for the quantification of cocrystals in the crystallization mixture. Drug Dev Ind Pharm 2012;38:923-9.

42. Vaghela P, Tank H, Jalpa P. Cocrystals: A novel approach to improve the physicochemical and mechanical properties. Indo Am J Pharm Res 2014:4:5055-65.

43. Fukte S, Wagh MP, Rawat S. Coformer selection: An important tool in cocrystal formation. Int J Pharm Pharm Sci 2014;6:9-14.

44. Cheney ML, Weyna DR, Shan N, Hanna M, Wojtas L, Zaworotko MJ. Coformer selection in pharmaceutical cocrystal development: A case study of a meloxicam aspirin cocrystal that exhibits enhanced solubility and pharmacokinetics. J Pharm Sci 2011;100:2172-81.

45. Najar AA, Azim Y. Pharmaceutical co-crystals: A new paradigm of crystal engineering. J Indian Inst Sci 2014;94:45-68.

46. Akhalwaya $\mathrm{S}$, van Vuuren $\mathrm{S}$, Patel $\mathrm{M}$. An in vitro investigation of indigenous South African medicinal plants used to treat oral infections. J Ethnopharmacol 2018;210:359-71.

47. Babu NJ, Sanphui P, Nangia A. Crystal engineering of stable temozolomide cocrystals. Chem Asian J 2012;7:2274-85

48. Aakeröy CB, Forbes S, Desper J. Using cocrystals to systematically modulate aqueous solubility and melting behavior of an anticancer drug. J Am Chem Soc 2009;131:17048-9.

49. Rajurkar V, Sunil N, Ghawate V. Tablet formulation and enhancement of aqueous solubility of efavirenz by solvent evaporation Co-crystal technique. Med Chem 2015;2:2161-444.

50. Shete AS, Khandagale VV, Murthy M, Yadav AV, Sakhre S, Doijad RC. Solid state characterization and tableting studies of ethanol based cocrystals of fenofibrate with nicotinamide. Indian J Pharm Educ Res 2018;51:71-7.

51. Oertling H, Besnard CL, Alzieu T, Wissenmeyer M, Vinay C, Mahieux J, et al. Ionic cocrystals of sodium chloride with carbohydrates. Cryst Growth Des 2016;17:262-70.

52. Duggirala NK, Perry ML, Almarsson Ö, Zaworotko MJ. Pharmaceutical 
cocrystals: Along the path to improved medicines. Chem Commun 2016;52:640-55

53. Batisai E, Ayamine A, Kilinkissa OE, Bathori N. Melting pointsolubility-structure correlations in multicomponent crystal containing fumaric or adipic acid. Cryst Eng Comm 2014;16:9992-8.

54. Stanton MK, Bak A. Physicochemical properties of pharmaceutical cocrystals: A case study of ten AMG 517 cocrystals. Cryst Growth Des 2008;8:3856-62.

55. Aakeröy CB, Forbes S, Desper J. Using cocrystals to systematically modulate aqueous solubility and melting behavior of an anticancer drug. J Am Chem Soc 2009;131:17048-9.

56. Fleischman SG, Kuduva SS, McMahon JA, Moulton B, Walsh B, Rodriguez-Hornedo RD, et al. Crystal engineering of the composition of pharmaceutical phases: Multiple-component crystalline solids involving carbamazepine. Cryst Growth Des 2003;3:909-19.

57. Maeno Y, Fukami T, Kawahata M, Yamaguchi K, Tagami T, Ozeki T, et al. Novel pharmaceutical cocrystal consisting of paracetamol and trimethylglycine, a new promising cocrystal former. Int $\mathrm{J}$ Pharm 2014;473:179-86.

58. Zhou Z, Li W, Sun WJ, Lu T, Tong HH, Sun CC, et al. Resveratrol cocrystals with enhanced solubility and stability. Int J Pharmacol 2016;509:391-9.

59. Krishna GR, Shi L, Bag PP, Sun CC, Reddy CM. Correlation among crystal structure, mechanical behaviour and stability in cocrystals of vanillin isomers. Cryst Growth Des 2015;15:1827-32.

60. Pawan Preet S, Chadha R. Crystal structure prediction in the context of pharmaceutical polymorph screening and putative polymorphs of ciprofloxacin. Int J Pharm Pharm Sci 2017;9:1-9. 